DOI: $10.20472 /$ IAC.2019.049.023

\title{
MISAKI KOHAMA
}

Aoyama Gakuin University, Japan

KEITA NISIO

Aoyama Gakuin University, Japan

IKUYA TAKAO

Aoyama Gakuin University, Japan

TAKASHI KABURAGI

International Chirstian University, Japan

TOSHIYUKI MATSUMOTO

Aoyama Gakuin University, Japan

SATOSHI KUMAGAI

Aoyama Gakuin University, Japan

YOSUKE KURIHARA

Aoyama Gakuin University, Japan

\section{UNCONSTRAINED APNEA DETECTION SYSTEM UTILIZING COMPRESSED TIME-FREQUENCY DISTRIBUTION BY AN AUTOENCODER}

\begin{abstract}
:
Currently, ischemic heart disease is the most common cause of death worldwide, with 9.48 million people dying of the disease. More than $30 \%$ of ischemic heart disease patients have sleep apnea syndrome. Since it is difficult to detect whether a patient has sleep apnea syndrome; the consultation rate remains at $15 \%$. Hence, routine monitoring is necessary to identify suspected patients. Many kinds of research on apnea detection have been conducted so far. The authors have also proposed a pneumatic method to measure a respiration signal unconstrainedly. In this study, we propose an unconstrained system to detect each occurrence of an apnea event by applying an autoencoder with two layers to the respiration signal.

In the proposed method, we measure a signal from a pressure sensor placed under a mattress on which a person is lying. To obtain the respiration signal, a digital band-pass filter with cut-off frequencies ranging from 0.15 to $0.45 \mathrm{~Hz}$ is applied to the output signal from the pressure sensor. Wavelet transform is applied to the filtered respiration signal to obtain time-frequency distributions that represent frequencies characteristic for each time step. Then, the time-frequency characteristic sends inputs to an autoencoder with two layers and the dimensions of the time-frequency distribution is compressed. The proposed method consists of two phases: a training phase and a detection phase. In the training phase, the compressed data with supervised data that is expressed as 0 or 1(0: respiration, 1: apnea) is used to train a classification model including softmax function. In the detection phase, to detect an apnea event, the compressed data without supervised data is applied to the trained classification model.

In the verification experiment, we prepared five subjects with variations in height and weight and acquired each subject's data in four recumbent positions. Data obtained was a total of 20 (five
\end{abstract}


subjects $x$ four recumbent positions). The dimension of time-frequency distribution was set to 113 . In the first layer, the dimension was compressed to 75 dimensions; and in the second layer, 75 dimensions were compressed to 50 . The performance of the proposed method is evaluated by the leave-one-subject-out and leave-one-recumbent-position-out cross-validations.

Based on results of cross-validations on all 20 data, the average correct answer rate was 0.90 for both the subjects and the recumbent positions. These results demonstrate that the proposed system was not susceptible to the subject and the recumbent position.

\section{Keywords:}

sleep apnea, time-frequency distribution, autoencoder, wavelet transform

JEL Classification: 119 